\section{Basiswissen zur Gutachtenerstellung}

$\mathrm{D}$ as knapp 300 Seiten umfassende Werk ist in vier Teile gegliedert. Es beginnt mit „Allgemeinen Grundlagen und $\mathrm{Zu}$ standsbegutachtung I“. Hier finden sich Kapitel zu allgemeinen und rechtlichen Grundlagen der Begutachtung, Erstellung von Gutachten, Begutachtung der Leistungsfähigkeit, Rehabilitation und Schwerbehindertenrecht. Im zweiten Teil widmen sich die Autoren der „Kausalitätsbezogenen Begutachtung“. Neben Beiträgen zu Kausalitäts- und Beweisregeln im Straf-, Zivilund Sozialrecht, zur Rolle von Haftpflicht-, gesetzlicher und privater Unfallversicherung finden sich hier auch Kapitel zum sozialen Entschädigungsrecht und Arzt- haftpflichtrecht. Im dritten Teil „Zustandsbegutachtung II" werden Pflegeversicherung, private Kranken-, Krankentagegeldund Berufsunfähigkeitsversicherung behandelt. Am Ende eines jeden Kapitels sind Gutachtenbeispiele aufgeführt und kommentiert. Der letzte Teil des Buches widmet sich „speziellen Begutachtungsfragen“" (u.a. Besonderheiten der psychiatrischen und forensischen Begutachtung, Begutachtung von Migranten, Begutachtung von Schmerzsyndromen sowie medizinische Begutachtung aus richterlicher Sicht).

Als Einstiegsliteratur kann das vorliegende Buch vorbehaltlos jedem empfohlen werden, der sich allgemein mit gut-

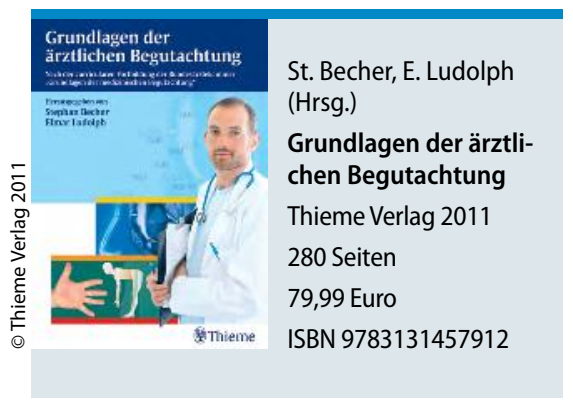

achterlichen Fragestellungen befassen möchte oder muss. Die wichtigsten Grundlagen der ärztlichen Begutachtung werden verständlich vermittelt, der Leser wird mit Termini und Denkansätzen vertraut gemacht, die für eine seriöse Gutachtenerstellung unabdingbar sind.

Prof. Dr. Gerhard Grevers

\title{
Konktrete Fallbeispiele der Rhinoplastik
}

$m$ ersten Teil des Buches, der etwa ein Fünftel des Gesamtumfangs ausmacht, gibt der Herausgeber einleitende Hinweise zur Vorbereitung der Operation, Op-Techniken, Nachsorge und Komplikationsmöglichkeiten. Der überwiegende Teil des Buches widmet sich dann - reichlich bebildert und mit exzellenten Zeichnungen versehen - konkreten Fallvorstellungen und deren Lösungsmöglichkeiten. Dabei haben zahlreiche Autoren mitgewirkt, die ihren Zugang zu vorgegebenen Fragestellungen anhand eigener Fälle aufzeigen. Jeder „case“ ist prä- und postoperativ dokumentiert, die individuelle Vorgehensweise anhand von
Zeichnungen und intraoperativen Bildern festgehalten. Der Leser kann im „nasal deformities index" am Ende des Buches, in dem alle Veränderungen in alphabetischer Reihenfolge aufgeführt sind, gezielt nach der gewünschten Fragestellung suchen und findet in der Regel mehrere Fallbeispiele, die von den einzelnen Chirurgen auch durchaus unterschiedlich angegangen werden. Hier lässt sich sehr gut verfolgen, dass auch in der Rhinoplastik nicht nur ein Weg nach Rom führt!

Auch wenn nicht alle Operationsergebnisse wirklich überzeugen können und die Intervalle zwischen der prä- und post-

\section{Praxisnahes Schwindel-Kompendium}

$Z^{\mathrm{s}}$ ehn Jahre nach Erscheinen der Erstauflage legen die Herausgeber, weit über die „Schwindelszene“ hinaus bekannte Experten vom Deutschen (sic!) Schwindelzentrum, Campus Großhadern in München, eine vollständig überarbeitete Fassung zum Thema vor. Nach einer Einführung zu Untersuchungsgang und apparativen Methoden werden in fünf weiteren Kapitel die verschiedenen klinischen Erscheinungsbilder unter Berücksichtigung neuer Erkenntnisse zu Epidemiologie, Pathophysiologie, Diagnostik und Therapie systematisch bearbeitet. Ob wirklich alles so neu ist, mag dahingestellt bleiben - zumindest dem HNO-Arzt sollten die im Vorwort besonders hervorgehobene Kortikosteroidtherapie bei akuter Neuritis vestibularis oder die Hochdosislangzeitbehandlung mit Betahistin bei M. Meniere bereits zur Facharztprüfung geläufig sein. Dies mindert den Stellenwert des Buches jedoch keineswegs, da alles Wichtige zum Thema kurz und prägnant, gleichzeitig aber übersichtlich und verständlich angesprochen wird. Hier wird umfangreiches Wissen aus jahrezehntelanger patientennaher Erfahrung in einem der großen Schwindelzentren der Repu-

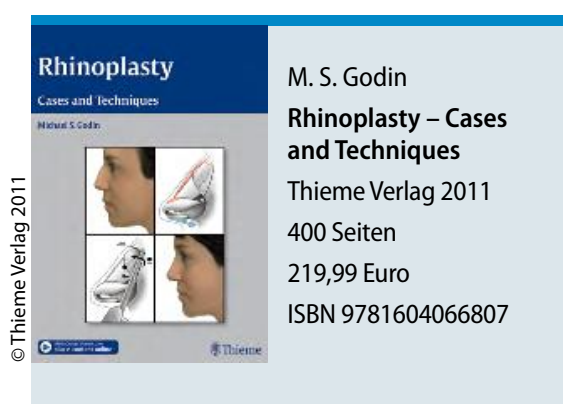

operativen Dokumentation zum Teil nur vier bis sechs Monate betragen, so ist hier doch ein gelungenes Buch zum Thema Rhinoplastik entstanden, das eine Menge praktisch-nützlicher Hinweise bereithält.

Prof. Dr. Gerhard Grevers 